\title{
ACTIVE VIBRATION CONTROL OF SMART PIEZO CANTILEVER BEAM USING PID CONTROLLER
}

\author{
Saurabh Kumar ${ }^{1}$, Rajeev Srivastava ${ }^{2}$, R.K.Srivastava ${ }^{3}$ \\ ${ }^{1}$ Research Scholar, Motillal Nehru National Institute of Technology, Allahabad - 211004, Uttar Pradesh, INDIA, \\ ${ }^{2}$ Associate Professor, Motillal Nehru National Institute of Technology, Allahabad - 211004, Uttar Pradesh, INDIA, \\ ${ }^{3}$ Professor, Motillal Nehru National Institute of Technology, Allahabad - 211004, Uttar Pradesh, INDIA,
}

\begin{abstract}
In this paper the modelling and Design of a Beam on which two Piezoelectric Ceramic Lead Zirconate Titanate ( PZT) patches are bonded on the top and bottom surface as Sensor/Actuator collocated pair is presented. The work considers the Active Vibration Control (AVC) using Proportional Integral Derivative (PID) Controller. The beam is assumed as Euler-Bernoulli beam. The two PZT patches are also treated as Euler-Bernoulli beam elements. The contribution of mass and stiffness of two PZT patches in the design of entire structure are also considered. The beam is modelled using three Finite Elements. The patches can be bonded near the fixed end, at middle or near the free end of the beam as collocated pair. The design uses first two dominant vibratory modes. The effect of PZT sensor/actuator pair is investigated at different locations of beam in vibration control. It can be concluded from the work that best result is obtained when the PZT patches are bonded near the fixed end.
\end{abstract}

Keywords: Smart Beam, Active Vibration control, Piezoelectric, PID Controller, Finite Element

\section{INTRODUCTION}

Active vibration control is a technique in which the vibration of a structure is controlled by applying counter force to the structure that is appropriately out of phase but equal in amplitude to the original force. As a result two opposite forces cancel each other and structure stops vibrating. Piezoelectric and Piezoelectric Ceramic materials can be used as sensors and actuators. These materials have ability to transform mechanical energy to electrical energy and vice-versa. A piezoelectric material is a crystal in which electricity is produced by pressure (Direct Effect). Conversely, a piezoelectric material deforms when it is subjected to an electric field (Converse Effect). The piezoelectric sensor senses the external disturbances and generates voltage due to direct piezoelectric effect while piezoelectric actuator produces force due to converse piezoelectric effect which can be used as controlling force. For generating the appropriate controlling force according to the sensed signal controller is needed. Tran Ich Thinh, Le Kim Ngoc [1] has developed a Finite Element (FE) model based on the First-Order Shear Deformation Theory for the static flexural shape and vibration control of a glass fiber/polyester composite plate bonded with piezoelectric actuator and sensor patches. The piezoelectric mass and stiffness are taken into account in the model. The results obtained were in good agreement with actual experimental result. Aydin Azizi, Laaleh Durali, Farid Parvari Rad, Shahin Zareie [2] used PZT elements as sensors and actuator to control the vibration of a cantilever beam. They studied the effect of different types of controller on vibration control. Finite Element Analysis and generalized equation of motion has been used in this paper. Premjyoti G.Patil [3] provides a mathematical model for the deformation of cantilever beam using Finite Element Method. Using the mathematical model, the beam deformation is plotted using MATLAB. Lucy Edery-Azulay, Haim Abramovich [4] described that the active damping is obtained by using an actuator and a sensor piezoceramic layer acting in closedloop. By transferring the accumulated voltage on the sensor layer to the piezoelectric actuator layer, the beam can actively damp-out its vibrations. An exact mathematical model, based on a first order shear deformation theory (FSDT) is developed and described. This model allows the investigation of piezo-composite beams with two actuation/sensing type mechanisms, extension and shear. For obtaining the natural frequency and mode shapes expressions were programmed in Maple 9. Effect of different piezoelectric materials on damping was also studied in this paper. Using the Euler-Bernoulli Beam Theory R. Ly, M. Rguiti, S. D'Astorg, A. Hajjaji, C. Courtois, and A. Leriche [5] developed a model of piezoelectric cantilever beam. The equations of motion for the global system were established using Hamilton's principle and solved using the modal decomposition method which described dynamic behaviour of the beam for energy harvesting. Then the model was implemented using MATLAB software and will be able to integrate with the circuit model for energy storage. The results obtained show a good agreement with the experiments and other previous works. Magdalene Marinaki, Yannis Marinakis, Georgios E. Stavroulakis [6] focused on the design of a vibration control mechanism for a beam embedded with piezoelectric sensors 
and actuators. The modelling of the piezoelectric sensors and actuators are based on the piezoelectric constitutive equations and classical equations of motion using finite element analysis. One nature- inspired intelligence method, the Particle Swarm Optimization (PSO) which is a population based swarm intelligence algorithm is used for the vibration control of the beam. Three different variants of the Particle Swarm Optimization were tested, namely, the simple Particle Swarm Optimization, the inertia Particle Swarm Optimization and the Constriction Particle Swarm Optimization. The same problem has been solved with two other optimization algorithms, namely a Genetic Algorithm and a Differential Evolution and the results are compared. Result shows that sufficient vibration suppression can be achieved by means of PSO method. K Ramesh Kumar and S Narayanan [7] used a finite element method based on EulerBernoulli beam theory. Linear quadratic regulator (LQR) controller is used for controlling. The LQR performance is taken as the objective for finding the optimal location of sensor-actuator pairs. The problem is formulated as a multiinput multi-output (MIMO) model control problem. The discrete optimal sensor and actuator location problem is formulated in the framework of an optimization problem which is solved using genetic algorithms (GAs). The study of the optimal location of actuators and sensors is carried out for different boundary conditions of beams like cantilever, simply supported and clamped boundary conditions. Jingjun Zhang, Lili He, Ercheng Wang, Ruizhen Gao [8] takes a cantilever beam bonded with rectangular shaped piezoelectric sensors and actuators. Two active vibration control methods such as Linear Quadratic Gauss (LQG) optimal control and robust $\mathrm{H} \infty$ control are investigated. The paper demonstrates that compared with the LQG control method, $\mathrm{H} \infty$ control has strong robustness to modal parameters variation and has a good closed-loop dynamic performance. C.M.A. Vasques, J. Dias Rodrigues [9] used an analysis and comparison of the classical control strategies, constant amplitude and constant gain velocity feedback (CAVF and CGVF), and optimal control strategies, linear quadratic regulator (LQR) and linear quadratic Gaussian (LQG) controller in order to investigate their effectiveness to suppress vibrations in beams with piezoelectric patches acting as sensors or actuators. In the paper a three-layered smart beam with two piezoelectric surface layers were modelled. The transverse displacement time history, at the free end is evaluated with the open- and closed-loop classical and optimal control systems. The case studies allow the comparison of their performances demonstrating their advantages and disadvantages. T.C Manjunath and B Bandyopadhyay. [10] used multirate output feedback based discrete sliding mode control for SISO systems in vibration control of Timoshenko smart structure. The beam structure is divided in four Finite Elements. The beam structure is modelled in the State Space form using the concept of piezoelectric theory. The performance of structure is investigated for first two dominant vibratory modes as well as higher modes. The effect of placing the piezoelectric collocated pair is investigated at various locations on the beam. Michele Betti, Georgios E. Stavroulakis and Charalambos C.
Baniotopoulos [11] used an active vibration control technique for a smart beam. The structure is made of two layers of piezoelectric material (PZT8) embedded on the surface of an aluminium beam. Piezoelectric sensors and actuators are perfectly bonded on the host elastic structure. A Finite Element model for a composite smart beam was developed. The integration of control actions is done within the ANSYS. The ANSYS Parametric Design Language (APDL) is used in order to develop a closed loop feedback control law. The control law can be calculated from a classical control theory, e.g. a linear feedback and LQR, which is used in this paper. By taking into account the modal shape of the beam, it is possible to suggest the optimal position for piezoelectric patches.

Most of the present researchers have used Finite element Analysis with different control laws to suppress the vibration of a Piezoelectric Smart Structure. The objective of this work is to design and analyse Piezoelectric Smart Beam with commonly used control method, The Proportional Integral Derivative (PID) when Piezoelectric Sensor/Actuator pair is placed at different locations on the beam. The paper is organized in three parts i.e. FE formulation of Smart beam, Controller Design, and Results.

\section{MODELLING OF THE SMART CANTILEVER BEAM}

Consider an Aluminium Cantilever beam bonded with collocated Piezoelectric Sensor/Actuator pair. Properties of beam and PZT patches (Actuator and Sensor) are given in Table-1. An external disturbing force $\mathbf{F}_{\text {Dist }}$ is acting at the free end. $\mathbf{F}_{\text {Actu }}$ is the force generated by the Actuator. Fig- 1, 2 and 3 shows three positions of the PZT Sensor/Actuator pair. The beam is divided in 3 Finite Elements. The beam may be divided in more than 3 Finite Elements (say 4, 5, 6 etc.). The more Finite Elements we take the more accurate results we get. The beam is considered as Euler-Bernoulli Beam and PZT patches are also considered as EulerBernoulli beam elements. We first start with the modelling of regular beam element and then modelling of the Smart beam element i.e. element having Piezoelectric Sensor/Actuator pair also. Finally all the elements are assembled using FE analysis. In modelling and analysis the following assumptions are taken:-

- The beam, sensor and actuator are taken as EulerBernoulli beam elements i.e. effect of transverse shear forces is neglected.

- Sensor and actuator layers are thin compared with the beam thickness.

- Cross- sections of beam, sensor and actuator remain plane and normal to the deformed longitudinal axis before and also after bending.

- Neutral axis of beam, sensor and actuator passes through the centroid. 
- The polarization direction of the Sensor and actuator layers is in the thickness direction ( $\mathrm{z}$ axis)

- The electric field loading of the Sensor and actuator layers is uniform uniaxial in the $\mathrm{x}$-direction as shown in Figures :-1,2 and 3

- The piezoelectric material is homogeneous, transverse isotropic and elastic.

- Adhesive used in bonding the Sensor/Actuator does not contribute in mass and stiffness of Smart beam element.

In the present study suffix ' $b$ ' is used for regular beam element, suffix ' $p$ ' is used for PZT patch element, suffix ' $a$ ' is used for Actuator and suffix ' $\mathrm{s}$ ' is used for Sensor. $\mathrm{A}_{\mathrm{b}}$ and $A_{p}$ are cross sectional areas of regular beam and PZT patch elements respectively. $I_{b}$ and $I_{p}$ are moment of Inertias of regular beam and PZT patch elements respectively.

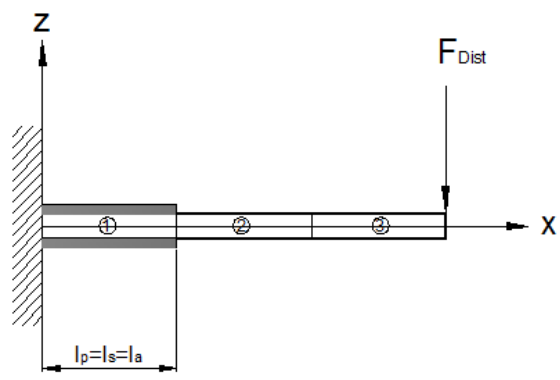

2 and 3: Beam Elements

1: Smart Beam Element

Fig:-1 Sensor/Actuator placed near Fixed End of the Beam
Table- 1: GEOMETRIC AND MATERIAL PROPERTIES OF BEAM AND PZT PATCHES

\begin{tabular}{|c|c|c|}
\hline$\underline{\text { PARAMETERS }}$ & $\frac{\frac{\text { CANTILEVER }}{\text { ALUMINUM }}}{\underline{\text { BEAM }}}$ & $\frac{\frac{\text { PIEZOELECTRIC }}{\text { SENSOR/ }}}{\underline{\text { ACTUATOR }}}$ \\
\hline Length & $l_{b}=0.3 \mathrm{~m}$ & $l_{p}=l_{a}=l_{s}=0.1 \mathrm{~m}$ \\
\hline Width & $b_{b}=0.03 \mathrm{~m}$ & $\mathrm{~b}_{\mathrm{p}}=\mathrm{b}_{\mathrm{a}}=\mathrm{b}_{\mathrm{s}}=0.03 \mathrm{~m}$ \\
\hline Thickness & $\mathrm{t}_{\mathrm{b}}=0.003 \mathrm{~m}$ & $\begin{array}{c}\mathrm{t}_{\mathrm{p}}=\mathrm{t}_{\mathrm{a}}=\mathrm{t}_{\mathrm{s}}=0.6 \times 10^{-3} \\
\mathrm{~m}\end{array}$ \\
\hline $\begin{array}{l}\text { Young's Modulus } \\
\text { of Elasticity }\end{array}$ & $\begin{array}{c}E_{b}=6.9 \times 10^{10} \\
N / m^{2}\end{array}$ & $\begin{array}{c}\mathrm{E}_{\mathrm{p}}=\mathrm{E}_{\mathrm{a}}=\mathrm{E}_{\mathrm{s}}=6.66 \times 10^{10} \\
\mathrm{~N} / \mathrm{m}^{2}\end{array}$ \\
\hline Density & $\rho_{\mathrm{b}}=2700 \mathrm{~kg} / \mathrm{m}^{3}$ & $\begin{array}{c}\rho_{\mathrm{p}}=\rho_{\mathrm{a}}=\rho_{\mathrm{s}}=7400 \\
\mathrm{~kg} / \mathrm{m}^{3}\end{array}$ \\
\hline $\begin{array}{c}\text { Piezoelectric } \\
\text { ( PZT-5H ) Stress } \\
\text { Constant }\end{array}$ & & $\mathrm{g}_{31}=8.5 \times 10^{-3} \mathrm{Vm} / \mathrm{N}$ \\
\hline $\begin{array}{c}\text { Piezoelectric } \\
\text { ( PZT-5H ) Strain } \\
\text { Constant }\left(\mathrm{d}_{31)}\right.\end{array}$ & & $\mathrm{d}_{31}=265 \times 10^{-12} \mathrm{C} / \mathrm{N}$ \\
\hline $\begin{array}{c}\text { Damping } \\
\text { Constants used }\end{array}$ & $\begin{array}{l}\alpha=0.001 \text { and } \\
\beta=0.0001\end{array}$ & \\
\hline
\end{tabular}

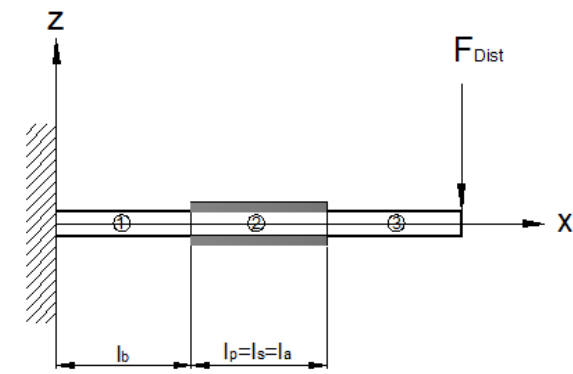

1and 3: Beam Elements

2: Smart Beam Element

Fig:-2 Sensor/Actuator placed at Middle of the Beam

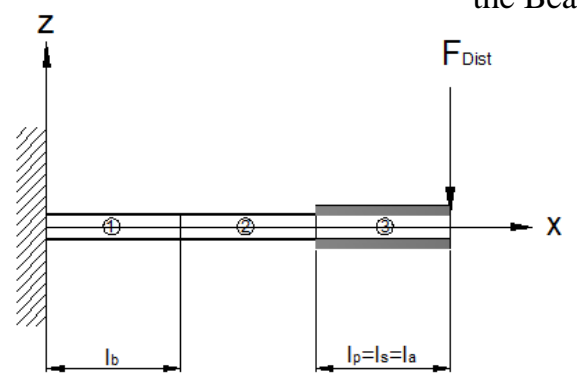

1 and 2: Beam Elements

3: Smart Beam Element

Fig:-3 Sensor/Actuator placed at Free End of the Beam 
A two nodes Finite element of a Smart Beam Element is shown in Fig-4
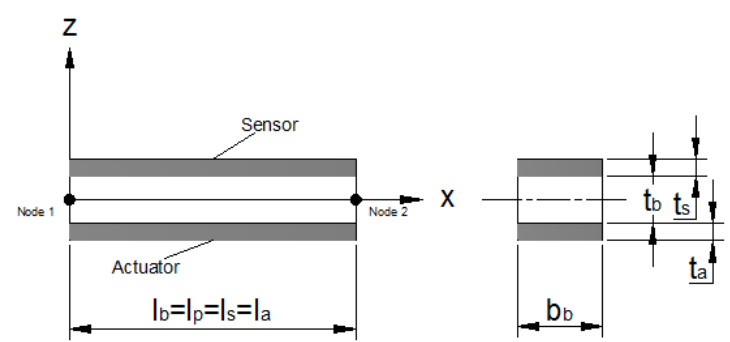

Fig-4 : A two nodes Finite element of a Smart Beam Element

\subsection{FE formulation of regular beam element}

A two nodes Finite element of a Regular Beam Element is shown in Fig-5

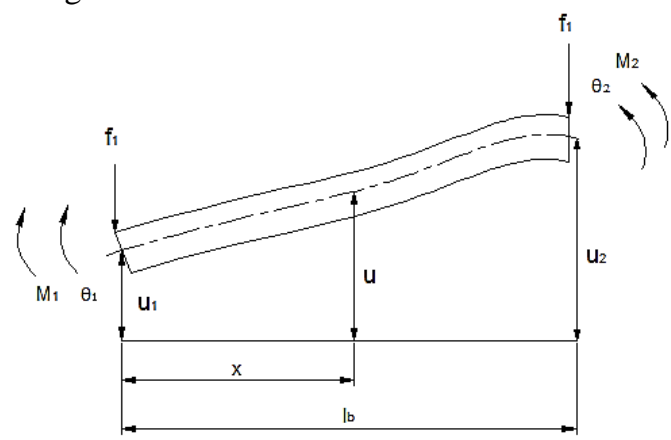

Fig-5 :A two nodes Finite element of a Regular Beam Element

The node undergoes both translational and rotational displacements and they are $\mathrm{u}_{1}, \theta_{1}, \mathrm{u}_{2}$ and $\theta_{2}$. The linear forces are $\mathrm{f}_{1}$ and $\mathrm{f}_{2}$ corresponding to linear displacements $\mathrm{u}_{1}$ and $\mathrm{u}_{2}$ and rotational joint forces i.e. Bending Moments are $\mathrm{M}_{1}$ and $\mathrm{M}_{2}$ corresponding to the rotational joint displacements $\theta_{1}$ and $\theta_{2}$. The Transverse displacement with in the element is assumed to be a cubic polynomial as

$u(x, t)=a_{1}+a_{2} x+a_{3} x^{2}+a_{4} x^{3}$

Substituting the boundary conditions the shape functions of beam elements can be obtained as

$$
\begin{aligned}
& \{N(x)\}^{T}=\left[\begin{array}{llll}
N_{1}(x) & N_{2}(x) & N_{3}(x) & N_{4}(x)
\end{array}\right]= \\
& {\left[1-\frac{3 x^{2}}{l_{b}^{2}}+\frac{2 x^{3}}{l_{b}^{3}} \quad x-\frac{2 x^{2}}{l_{b}}+\frac{x^{3}}{l_{b}^{2}} \quad \frac{3 x^{2}}{l_{b}^{2}}-\frac{2 x^{3}}{l_{b}^{3}} \quad \frac{-x^{2}}{l_{b}}+\frac{x^{3}}{l_{b}^{2}}\right]}
\end{aligned}
$$

The nodal displacement function can be written as

$$
\{q\}^{T}=\left[\begin{array}{llll}
u_{1} & \theta_{1} & u_{2} & \theta_{2}
\end{array}\right]
$$

The Lagrange's Equations gives the Kinetic Energy and Potential energy of the system respectively as :

$$
\begin{aligned}
& T=\frac{1}{2}\{\dot{q}\}^{T}[m]\{\dot{q}\} \\
& U=\frac{1}{2}\{q\}^{T}[k]\{q\}
\end{aligned}
$$

Using Lagrange's Equation the Element Stiffness Matrix and Mass Matrix of a beam element are computed that can be found in Text Books of Vibration [14], [15] and written as

$$
\begin{gathered}
{\left[k_{b}\right]=\frac{E_{b} I_{b}}{l_{b}^{3}}\left[\begin{array}{cccc}
12 & 6 l_{b} & -12 & 6 l_{b} \\
6 l_{b} & 4 l_{b}{ }^{2} & -6 l_{b} & 2 l_{b}^{2} \\
-12 & -6 l_{b} & 12 & -6 l_{b} \\
6 l_{b} & 2 l_{b}{ }^{2} & -6 l_{b} & 4 l_{b}^{2}
\end{array}\right]} \\
{\left[m_{b}\right]=\frac{\rho_{b} A_{b} l_{b}}{420}\left[\begin{array}{cccc}
156 & 22 l_{b} & 54 & -13 l_{b} \\
22 l_{b} & 4 l_{b}^{2} & 13 l_{b} & -3 l_{b}^{2} \\
54 & 13 l_{b} & 156 & -22 l_{b} \\
-13 l_{b} & -3 l_{b}^{2} & -22 l_{b} & 4 l_{b}^{2}
\end{array}\right]}
\end{gathered}
$$

The First, Second spatial derivatives of shape functions are denoted as under that will be used in deriving the Sensor and Actuator results.

$$
\begin{aligned}
& \left\{n_{1}(x)\right\}=\left\{\frac{d N(x)}{d x}\right\}=\left\{N^{\prime}(x)\right\} \text { and } \\
& \left\{n_{2}(x)\right\}=\left\{\frac{d^{2} N(x)}{d x^{2}}\right\}=\left\{N^{\prime \prime}(x)\right\}
\end{aligned}
$$

\subsection{FE formulation of smart beam element}

When PZT patches are assumed as Euler-Bernoulli beam elements the Elemental mass and stiffness matrices of PZT beam element can be computed in similar fashion as:

$\left[k_{p}\right]=\frac{E_{p} I_{p}}{l_{p}^{3}}\left[\begin{array}{cccc}12 & 6 l_{p} & -12 & 6 l_{p} \\ 6 l_{p} & 4 l_{p}{ }^{2} & -6 l_{p} & 2 l_{p}{ }^{2} \\ -12 & -6 l_{p} & 12 & -6 l_{p} \\ 6 l_{p} & 2 l_{p}{ }^{2} & -6 l_{p} & 4 l_{p}{ }^{2}\end{array}\right]$

$\left[m_{p}\right]=\frac{\rho_{p} A_{p} l_{p}}{420}\left[\begin{array}{cccc}156 & 22 l_{p} & 54 & -13 l_{p} \\ 22 l_{p} & 4 l_{p}^{2} & 13 l_{p} & -3 l_{p}^{2} \\ 54 & 13 l_{p} & 156 & -22 l_{p} \\ -13 l_{p} & -3 l_{p}^{2} & -22 l_{p} & 4 l_{p}^{2}\end{array}\right]$

The smart beam element is obtained by sandwiching the regular beam element in between the two PZT patches as shown in Fig.:-6

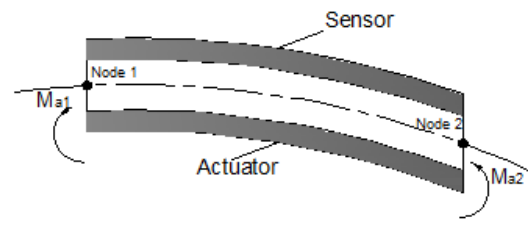

Fig-6 :A two nodes Finite element of a Smart Beam Element 
In which $E I=E_{b} I_{b}+2 E_{p} I_{p}$ is the Flexural rigidity and $\rho A=b_{b}\left(\rho_{b} t_{b}+2 \rho_{p} t_{p}\right)$ is the mass per unit length of smart beam element, $t_{P}$ is the thickness of PZT patches i.e. thickness of Actuator and Sensor. So the Elemental mass and stiffness matrices of Smart beam element are

$[k]=\frac{E I}{l_{p}^{3}}\left[\begin{array}{cccc}12 & 6 l_{p} & -12 & 6 l_{p} \\ 6 l_{p} & 4 l_{p}{ }^{2} & -6 l_{p} & 2 l_{p}{ }^{2} \\ -12 & -6 l_{p} & 12 & -6 l_{p} \\ 6 l_{p} & 2 l_{p}{ }^{2} & -6 l_{p} & 4 l_{p}{ }^{2}\end{array}\right]$

$[m]=\frac{\rho A l_{p}}{420}\left[\begin{array}{cccc}156 & 22 l_{p} & 54 & -13 l_{p} \\ 22 l_{p} & 4 l_{p}^{2} & 13 l_{p} & -3 l_{p}^{2} \\ 54 & 13 l_{p} & 156 & -22 l_{p} \\ -13 l_{p} & -3 l_{p}^{2} & -22 l_{p} & 4 l_{p}^{2}\end{array}\right]$

\section{SENSOR EQUATION}

Following Linear Piezoelectric Constitutive equations [6],[13] will be used for deriving the Sensor and Actuator equations.

$$
\begin{aligned}
& \varepsilon_{x}=S_{11}^{E} \sigma_{x}+d_{31} E_{z} \\
& \mathrm{D}_{z}=d_{31} \sigma_{x}+\xi_{33}{ }^{\sigma} E_{z}
\end{aligned}
$$

Where $\varepsilon$ is Strain, $\sigma$ is Stress, $S^{\mathrm{E}}$ is Compliance when electric field is constant, $d_{31}$ is Piezoelectric Constant (Coulomb/N or $\mathrm{m} / \mathrm{V}$ ), E is Electric field (Volt/m), D is Electric displacement i.e. charge per unit area $\left(\mathrm{Coulomb} / \mathrm{m}^{2}\right), \xi^{\sigma}$ is Dielectric constant ( Permittivity) under constant stress. The direct piezoelectric effect is used to calculate the output charge on the sensor layer created by the strains in the beam. Since no electric field is applied to the sensor layer, we get

$$
D_{z}=C_{11} d_{31} \varepsilon_{x}
$$

Where $\mathrm{C}_{11}$ is the Young's modulus of elasticity (Inverse of compliance).

The charge measured through the electrodes of the sensor is given by

$$
q(t)=\int_{S} D_{z} d s
$$

The current on the surface of the sensor is given by

$i(t)=\frac{d q(t)}{d t}$

From the Text books of Mechanics of Solids we know that strain at a point in a beam is given as $\varepsilon_{\mathrm{x}}=\mathrm{z} \mathrm{d}^{2} \mathrm{u} / \mathrm{dx}^{2}$, where $\mathrm{z}$ is a coordinate on the beam w.r.t. neutral axis. Width $\mathrm{b}_{\mathrm{b}}=\mathrm{b}_{\mathrm{s}}=\mathrm{b}_{\mathrm{a}}$. As such current generated can be written as [10]

$$
i(t)={ }_{z} C_{11} d_{31} b_{b} \int_{0}^{l_{p}}\left\{n_{2}(x)\right\}^{T}\{\dot{q}\} d x
$$

Where $\mathrm{z}=\mathrm{t}_{\mathrm{b}} / 2+\mathrm{t}_{\mathrm{s}}$ for maximum strain.
Voltage generated by the sensor is

$$
V^{S}(t)=G_{S} i(t)
$$

Where $\mathrm{G}_{\mathrm{S}}$ is the gain of the signal conditioning device

$$
V^{s}(t)=G_{s} C_{11} d_{31} z b_{b}\left[\begin{array}{llll}
0 & -1 & 0 & 1
\end{array}\right]\left[\begin{array}{llll}
\dot{u}_{1} & \dot{\theta}_{1} & \dot{u}_{2} & \dot{\theta}_{2}
\end{array}\right]^{T}
$$

This can be written as

$V^{s}(t)=C_{s}\left[\begin{array}{llll}0 & -1 & 0 & 1\end{array}\right]\{\dot{q}\}$

Where $C_{s}=G_{s} C_{11} d_{31} z b_{b}$ is Sensor Constant. The above equation can be written as

$V^{s}(t)=\{g\}^{T}\{\dot{q}\}$

Where $\{g\}$ is a Constant Vector of size $(4 \times 1)$

\section{ACTUATOR EQUATION}

From equation 12 the stress developed in the Actuator is

$$
\sigma_{x}=C_{11} d_{31} E_{z}
$$

Where $\mathrm{E}_{\mathrm{z}}$ is the Electric Field.

The resultant bending moment produced by the actuator is given by [10]

$$
M_{a}=C_{11} d_{31}\left(\frac{t_{a}+t_{b}}{2}\right) V^{a}(t)
$$

Where $\mathrm{V}^{\mathrm{a}}(\mathrm{t})$ is the voltage applied on the actuator which is given by

$$
V^{a}(t)=\text { Controller gain } \times V^{S}(t)
$$

The force produced by the Actuator is given by

$$
\left\{F_{\text {Actu }}\right\}=C_{11} d_{31} b_{b}\left(\frac{t_{a}+t_{b}}{2}\right) \int_{l_{a}} V^{a}(t)\left\{n_{1}(x)\right\} d x
$$

This can also be expressed as

$$
\left\{F_{\text {Actu }}\right\}=\{H\} V^{a}(t)
$$

Where $\{H\}$ is a constant vector of size $(4 \times 1)$ and is given as

$$
\{H\}^{T}=C_{11} d_{31} b_{b}\left(\frac{t_{a}+t_{b}}{2}\right)\left[\begin{array}{llll}
-1 & 0 & 1 & 0
\end{array}\right]
$$

or

$$
\{H\}^{T}=C_{a}\left[\begin{array}{llll}
-1 & 0 & 1 & 0
\end{array}\right]
$$

Where $\mathrm{C}_{\mathrm{a}}$ is the Actuator Constant and is given by

$$
C_{a}=C_{11} d_{31} b_{b}\left(\frac{t_{a}+t_{b}}{2}\right)
$$

\section{CONTROL LAW USING PID CONTROLLER}

Next goal is to achieve an appropriate controlled voltage that can be fed to the Actuator, for that a PID controller is 
used in the present study. A typical PID control law that can be used for Active Vibration Control is:

$$
y(t)=K_{p}+K_{i} \int e(t) d t+K_{d} \dot{e}(t)
$$

Where $\mathrm{y}(\mathrm{t})$ is control signal, $\mathrm{K}_{\mathrm{p}}, \mathrm{K}_{\mathrm{i}}$ and $\mathrm{K}_{\mathrm{d}}$ are Proportional, Integral and Derivatives gains respectively and $\mathrm{e}(\mathrm{t})$ and $\dot{\mathrm{e}}(\mathrm{t})$ are error signal and its derivative respectively. These three gains can be tuned in order to provide fine control for the application. For fine tuning of the controller the following

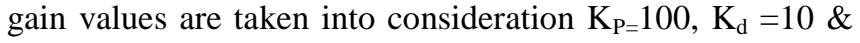
$\mathrm{K}_{\mathrm{i}}=50$.

A typical PID controller is shown in Fig.- 7

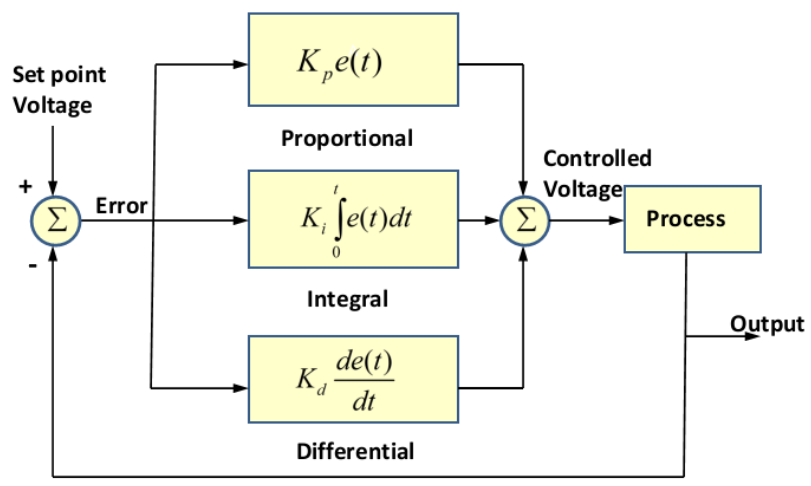

Fig-7 :A Typical PID Controller

\section{DYNAMIC EQUATION OF SMART STRUCTURE}

Next step is to formulate and solve the Equation of motion of entire structure that is given by

$[M]\{\ddot{q}\}+[K]\{\dot{q}\}=\left\{F_{\text {Dist }}\right\}+\left\{F_{\text {Actu }}\right\}$

Consider a generalized coordinate using a transformation

$\{q\}=[\psi]\{x\}$ for the first two dominant vibratory modes then equation of motion becomes

$\left[M^{r}\right]\{\ddot{x}\}+\left[K^{r}\right]\{x\}=\left\{F_{\text {Dist }}^{r}\right\}+\left\{F_{\text {Actu }}^{r}\right\}$

If damping of the structure is also considered then assuming proportional damping as

$[C]=\alpha[M]+\beta[K]$

The generalized dynamic equation of motion is given as

$\left[M^{r}\right]\{\ddot{x}\}+\left[C^{r}\right]\{\dot{x}\}+\left[K^{r}\right]\{x\}=\left\{F_{\text {Dist }}^{r}\right\}+\left\{F_{\text {Actu }}^{r}\right\}$

\section{STATE SPACE FORMULATION FOR THE FIRST TWO DOMINANT VIBRATION MODES}

Let the $\{\mathrm{x}\}=\{\mathrm{y}\}$ as

$$
\{x\}=\left[\begin{array}{l}
x_{1} \\
x_{2}
\end{array}\right]=\{y\}=\left[\begin{array}{l}
y_{1} \\
y_{2}
\end{array}\right]
$$

And

$$
\{\dot{x}\}=\{\dot{y}\}=\left[\begin{array}{c}
\dot{y}_{1} \\
\dot{y}_{2}
\end{array}\right]=\left[\begin{array}{l}
y_{3} \\
y_{4}
\end{array}\right] \text { and }\{\ddot{x}\}=\{\ddot{y}\}=\left[\begin{array}{c}
\dot{y}_{3} \\
\dot{y}_{4}
\end{array}\right]
$$

Equation of motion now can be written as

$$
\begin{aligned}
& {\left[M^{r}\right]\left[\begin{array}{l}
\dot{y}_{3} \\
\dot{y}_{4}
\end{array}\right]+\left[C^{r}\right]\left[\begin{array}{l}
y_{3} \\
y_{4}
\end{array}\right]+\left[K^{r}\right]\left[\begin{array}{l}
y_{1} \\
y_{2}
\end{array}\right]} \\
& =\left\{F_{\text {Dist }}^{r}\right\}+\left\{F_{\text {Actu }}^{r}\right\}
\end{aligned}
$$

This can be simplified as

$$
\begin{aligned}
& {\left[\begin{array}{l}
\dot{y}_{3} \\
\dot{y}_{4}
\end{array}\right]=-\left[M^{r}\right]^{-1}\left[K^{r}\right]\left[\begin{array}{l}
y_{1} \\
y_{2}
\end{array}\right]-\left[M^{r}\right]^{-1}\left[C^{r}\right]\left[\begin{array}{l}
y_{3} \\
y_{4}
\end{array}\right]} \\
& +\left[M^{r}\right]^{-1}\left\{F_{\text {Dist }}^{r}\right\}+\left[M^{r}\right]^{-1}\left\{F_{\text {Actu }}^{r}\right\}
\end{aligned}
$$

The above equation can be written in State form as

$$
\begin{aligned}
& {\left[\begin{array}{l}
\dot{y}_{1} \\
\dot{y}_{2} \\
\dot{y}_{3} \\
\dot{y}_{4}
\end{array}\right]=\left[\begin{array}{cc}
{[0]} & {[I]} \\
-\left[M^{r}\right]^{-1}\left[K^{r}\right] & -\left[M^{r}\right]^{-1}\left[C^{r}\right]
\end{array}\right]\left[\begin{array}{l}
y_{1} \\
y_{2} \\
y_{3} \\
y_{4}
\end{array}\right]} \\
& +\left[\begin{array}{c}
\{0\} \\
{\left[M^{r}\right]^{-1}[\psi]^{T}\{H\}}
\end{array}\right] V^{a}(t)+\left[\begin{array}{c}
\{0\} \\
{\left[M^{r}\right]^{-1}[\psi]^{T}\{f\}}
\end{array}\right] u(t)
\end{aligned}
$$

Where $u(t)$ is the magnitude of external force and $\{f\}$ is the unit force vector. The sensor voltage is taken as output of the structure which can be written as

$$
V^{s}(t)=\left[\{0\} \quad\{g\}^{T}[\psi]\right]\left[\begin{array}{llll}
y_{1} & y_{2} & y_{3} & y_{4}
\end{array}\right]^{T}
$$

So the State Space Model of smart structure for the first two dominant vibratory modes is given by

$$
\begin{aligned}
& \{\dot{y}\}=[A]\{y(t)\}+[B] V^{a}(t)+[D] u(t) \\
& \text { and } \\
& V^{s}(t)=[E]^{T}\{y(t)\}+[F] V^{a}(t)
\end{aligned}
$$

Where

$$
\begin{aligned}
& {[A]=\left[\begin{array}{cc}
{[0]} & {[I]} \\
-\left[M^{r}\right]^{-1}\left[K^{r}\right] & -\left[M^{r}\right]^{-1}\left[C^{r}\right]
\end{array}\right],} \\
& {[B]=\left[\begin{array}{c}
\{0\} \\
{\left[M^{r}\right]^{-1}[\psi]^{T}\{H\}}
\end{array}\right],[D]=\left[\begin{array}{c}
\{0\} \\
{\left[M^{r}\right]^{-1}[\psi]^{T}\{f\}}
\end{array}\right],} \\
& {[E]=\left[\begin{array}{c}
\{0\} \\
\{g\}^{T}[\psi]
\end{array}\right] \text {, and }[F]=\text { Null Matrix }}
\end{aligned}
$$




\section{RESULTS}

The beam is divided in the three Finite elements. Time response of the structure is studied after bonding the Sensor/actuator pair at different locations on the beam say near the fixed end, at the middle and at the free end. The results are shown in Figures- 8,9 and 10 respectively

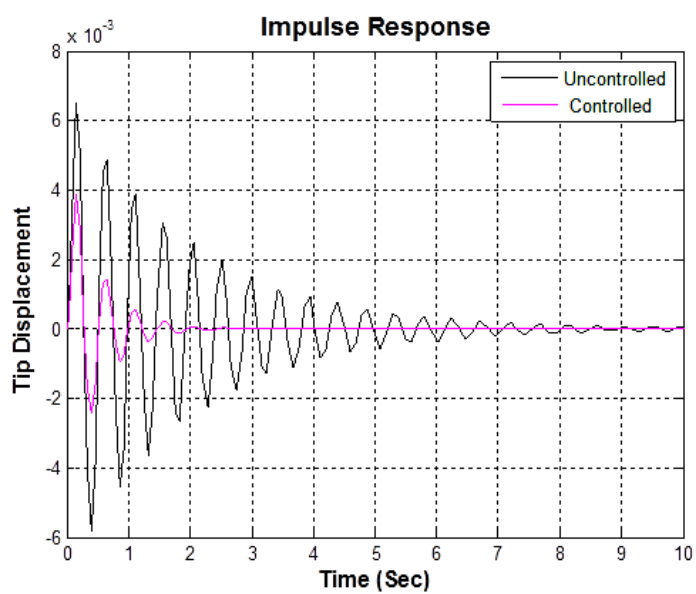

Fig- 8 : Tip Displacement of Beam when Sensor/Actuator pair is placed near Fixed End of the Beam

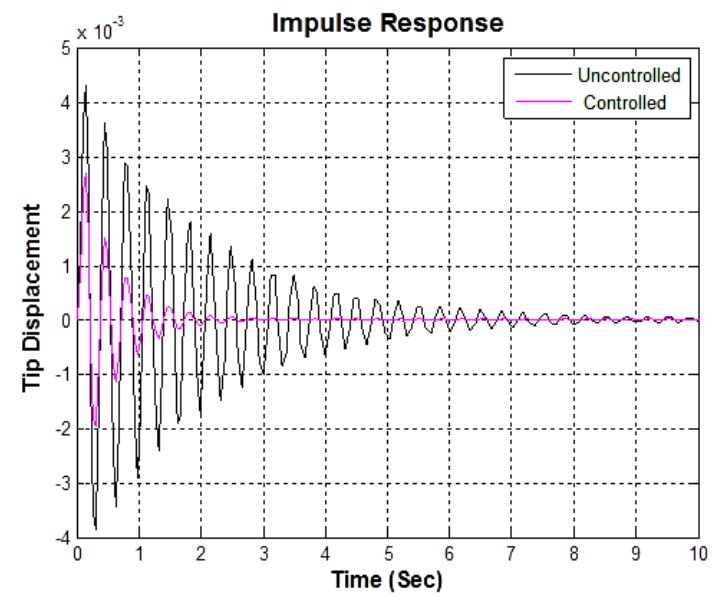

Fig- 9 : Tip Displacement of Beam when Sensor/Actuator pair is placed at Middle of the Beam

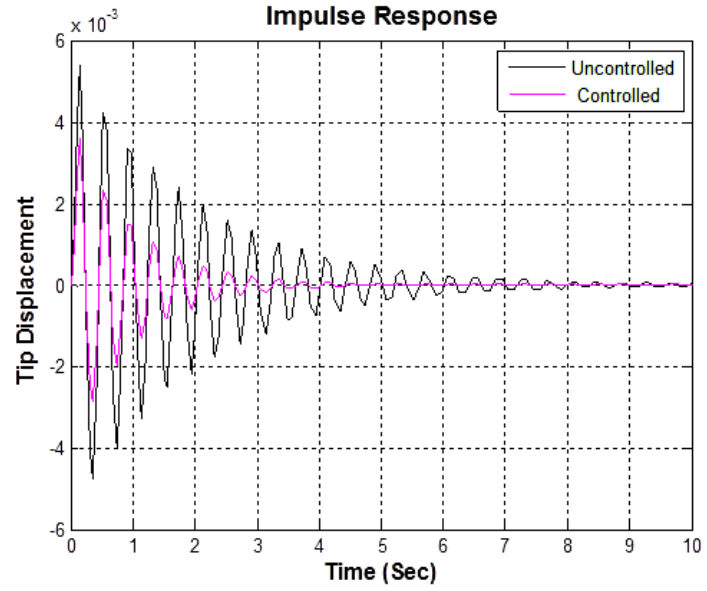

Fig- 10 : Tip Displacement of Beam when Sensor/Actuator pair is placed at Free End of the Beam

\section{CONCLUSIONS}

Present study is useful in controlling the vibration of modern day machines, Engineering structures, Automobiles, Gadgets Spacecrafts, Bridges, Marine equipments, Machine Tools, Off shore structures, High rise buildings etc. Present work deals with Active vibration control of a Cantilever Beam bonded with two Piezoelectric patches as collocated pair. It is observed that without control, response is paramount but after applying control force sufficient vibration suppression has been achieved. Results are taken after placing the Piezoelectric Actuator/Sensor Pair near fixed end, at middle and near free end of the beam. It can be concluded from the work that best result is obtained when the Piezoelectric patches are bonded near the fixed end.

\section{REFERENCES}

[1]. Tran Ich Thinh , Le Kim Ngoc , "Static behavior and vibration control of piezoelectric cantilever composite plates and comparison with experiments", Computational Materials Science 49 (2010) S276-S280

[2]. Aydin Azizi, Laaleh Durali, Farid Parvari Rad, Shahin Zareie, "Control of vibration suppression of a smart beam by piezoelectric elements", 2009 Second International Conference on Environmental and Computer Science, IEEE Computer Society

[3]. Premjyoti G.Patil, "An Efficient Model for Vibration Control by Piezoelectric Smart Structure Using Finite Element Method", European Journal of Scientific Research ISSN 1450-216X Vol.34 No.4 (2009), pp.485-494

[4]. Lucy Edery-Azulay , Haim Abramovich , "Active damping of piezo-composite beams", Composite Structures 74 (2006 458-466

[5]. R. Ly, M. Rguiti, S. D’Astorg, A. Hajjaji, C. Courtois, A. Leriche, "Modeling and characterization of piezoelectric cantilever bending sensor for energy harvesting", Sensors and Actuators A 168 (2011) 95-100

[6]. Magdalene Marinaki, Yannis Marinakis, Georgios E. Stavroulakis, "Vibration control of beams with piezoelectric sensors and actuators using particle swarm optimization", Expert Systems with Applications 38 (2011) 6872-6883

[7]. K Ramesh Kumar and S Narayanan, "Active vibration control of beams with optimal placement of piezoelectric sensor/actuator pairs", Smart Mater. Struct. 17 (2008) 055008

[8]. Jingjun Zhang, Lili He, Ercheng Wang, Ruizhen Gao, "Active Vibration Control of Flexible Structures Using Piezoelectric Materials",2008 International Conference on Advanced Computer Control, IEEE Computer Society

[9]. C.M.A. Vasques, J. Dias Rodrigues , "Active vibration control of smart piezoelectric beams: 
Comparison of classical and optimal feedback control strategies", Computers and Structures 84 (2006) 1402-1414

[10]. T.C. Manjunath, B. Bandyopadhyay, "Vibration control of Timoshenko smart structures using multirate output feedback based discrete sliding mode control for SISO systems" , Journal of Sound and Vibration 326 (2009) 50-74

[11]. Michele Betti, Georgios E. Stavroulakis and Charalambos C. Baniotopoulos, "Active vibration suppression of smart beams", PAMM - Proc. Appl. Math. Mech. 6, 799-800 (2006)

[12]. Sinan Korkmaz, "A review of active structural control: challenges for engineering informatics", Computers and Structures 89 (2011) 2113-2132

[13]. A. Preumont, "Vibration Control of Active Structures-An Introduction", 3rd Edition , Springer

[14]. William T. Thomson, "Theory of vibration with Applications" CBS Publishers \& Distributors

[15]. S.S. Rao, "Mechanical Vibrations" Addison Wesley Publishing Co. 